\title{
Early-Onset Neonatal Sepsis with Extended Spectrum Beta-Lactamase Producing Escherichia Coli in Infants Born to South and South East Asian Immigrants: A Case Series
}

\author{
Kalsang Dolma, MD ${ }^{1}$ Thanh L. Summerlin, MD ${ }^{1} \quad$ Hansa Wongprasert, MD ${ }^{1} \quad$ Charitharth Vivek Lal, MD 1 \\ Joseph B. Philips III, MD ${ }^{1}$ Lindy Winter, MD ${ }^{1}$ \\ ${ }^{1}$ Division of Neonatology, Department of Pediatrics, University of \\ Alabama at Birmingham, Birmingham, Alabama \\ Address for correspondence Kalsang Dolma, MD, Division of \\ Neonatology, Department of Pediatrics, University of Alabama at \\ Birmingham, 176F Suite 9380, Women and Infants Center, 619 South \\ Am J Perinatol Rep 2018;8:e277-e279. \\ 19th Street, Birmingham, AL 35249-7335 \\ (e-mail: kdolma@uabmc.edu).
}

\author{
Abstract \\ Keywords \\ - neonatal sepsis \\ - early onset sepsis \\ - extended-spectrum $\beta$ - \\ lactamases \\ - Escherichia coli
}

\begin{abstract}
Extended-spectrum $\beta$-lactamase (ESBL)-producing Enterobacteriaceae represent a major worldwide threat. We present three cases of early onset ESBL Escherichia coli sepsis in infants born to families from South and Southeast Asia to inform the practitioner community about this emerging threat. Infants with suspected sepsis, whose mother is from Asia or Southeast Asia, should be suspected of having an infection with an ESBL-producing organism, and practitioners should strongly consider adding a carbapenem to their usual initial antibiotic regimen.
\end{abstract}

Extended-spectrum $\beta$-lactamases (ESBL) are plasmid encoded enzymes produced by Enterobacteriaceae and induce bacterial resistance by hydrolyzing $\beta$-lactam antibiotics. ${ }^{1,2}$ ESBL-producing Enterobacteriaceae represent a major worldwide threat and contribute to morbidity and mortality among newborn infants, especially in other parts of the world with a pooled prevalence of $11 \%{ }^{3}$ In the U.S., early onset sepsis (EOS) in neonates with ESBL organisms is rare. We present three cases of EOS with ESBL Escherichia coli (E. coli) in infants born to mothers, recently immigrated from South and Southeast Asia.

\section{Case Series}

\section{Case 1}

A 34-week expected gestational age (EGA) male was born to a 32 years/o Pakistani mother via spontaneous vaginal delivery, with rupture of membranes 12 hours before delivery. Pregnancy was complicated by gestational diabetes and severe pre-eclampsia. The mother received two doses of betamethasone and magnesium sulfate. Apgar scores were 6 and 8 at 1 and 5 minutes, respectively. The infant initially did well and was transferred to a step down unit on day 2 . No antibiotics were administered during the initial Neonatal Intensive Care Unit (NICU) stay. At 62 hours of age, skin mottling, lethargy, abdominal distension, delayed capillary refill hypotension, and oliguria developed. A sepsis evaluation was initiated and ampicillin, gentamicin, and acyclovir were begun. Respiratory failure led to intubation and mechanical ventilation. Laboratory results revealed elevated $\mathrm{C}$ reactive protein $(89.9 \mathrm{mg} / \mathrm{L})$, leukopenia $(1,004 / \mu \mathrm{L})$, neutropenia $(100 / \mu \mathrm{L})$, and thrombocytopenia $(71,000 / \mu \mathrm{L})$, and severe metabolic acidosis with lactic acid of $16 \mathrm{mmol} / \mathrm{L}$. Multiple doses of sodium bicarbonate $\left(\mathrm{NaHCO}_{3}\right)$ and fluid resuscitation did not improve the acidosis. The infant developed shock refractory to fluid boluses and vasopressors. Blood culture grew gram-negative rods within 7 hours of draw, and cefepime was added for extended gram-negative coverage. Cerebrospinal fluid (CSF) results revealed gramnegative meningitis. The infant developed pulmonary hemorrhage from disseminated intravascular coagulation (DIC) within 24 hours of presentation and continued to receive multiple blood products without improvement. He rapidly deteriorated despite vigorous resuscitative efforts and died. Blood and CSF cultures grew ESBL E. coli. received

June 15, 2018 accepted after revision August 3, 2018
DOI https://doi.org/

10.1055/s-0038-1675336. ISSN 2157-6998.
Copyright $\odot 2018$ by Thieme Medical Publishers, Inc., 333 Seventh Avenue, New York, NY 10001, USA. Tel: +1(212) 584-4662.
License terms

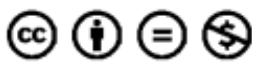




\section{Case 2}

A 35-week EGA male twin 2 was born to a 26 years/o Vietnamese mother after an uncomplicated pregnancy. Maternal Group B Streptococcus (GBS) status was unknown, and mother received two doses of ampicillin prior to delivery. Mother had no signs of chorioamnionitis. The well-appearing infant was admitted to the well-baby nursery. At 60 hours, he developed hypothermia and new onset apnea. Rectal temperature was $94^{\circ} \mathrm{F}$, and poor perfusion, and decreased activity were present. A sepsis evaluation was initiated and ampicillin, gentamicin, and ceftazidime were started. The infant was intubated with worsening respiratory status and persistent apnea. Pulmonary hemorrhage was noted during intubation. Chest X-ray showed near total opacification of the right lung. The baby was placed on conventional ventilation and multiple doses of endotracheal epinephrine were given without improvement. Multiple blood products were given for DIC. Blood gases showed a severe mixed acidosis. He deteriorated rapidly and died 4 hours after onset of symptoms. Blood culture grew ESBL E. coli.

\section{Case 3}

A 30-week EGA male infant was born to a 35 years/o Indian mother via spontaneous vaginal delivery. Pregnancy was complicated by gestational diabetes and prolonged rupture of membrane (48 hours). Maternal GBS status was unknown. She received four doses of ampicillin prior to delivery. There were no signs of chorioamnionitis; however, she developed a fever of $101.7^{\circ} \mathrm{F}$ on postpartum day 1 and was diagnosed with endometritis. Apgar scores were 7 and 8 at 1 and 5 minutes, respectively, and the infant was transferred to the NICU on room air. At 3 hours of age, he developed respiratory distress requiring continuous positive airway pressure (CPAP), which was escalated to conventional and then high frequency ventilation within the next 12 hours. Chest films showed generalized granular infiltration. Ampicillin, gentamicin, and ceftazidime were started. His status deteriorated with compensated septic shock, tachycardia, and lactic acidosis (11.6 mmol/L). DIC was present with pulmonary hemorrhage, thrombocytopenia, and coagulopathy. He received multiple normal saline boluses and blood products. Dopamine was added for hypotension. Blood culture grew gram-negative rods within 12 hours. Due to his ethnicity, meropenem was added due to high suspicion for ESBL gram-negative sepsis. Lumbar puncture was deferred due to unstable clinical status and significant coagulopathy (international normalized ratio [INR] of 4.1). Blood culture grew ESBL $E$. coli susceptible to meropenem and gentamicin, but resistant to all cephalosporins. Placental pathology revealed acute severe subchorioamnionitis. Given the severity of his illness with ESBL E.coli bacteremia and no initial CSF studies, he completed a 21day course of meropenem for presumed meningitis associated with his bacteremia and sepsis.

\section{Discussion}

ESBLs induce bacterial resistance by hydrolyzing penicillins, first, second, and third generation cephalosporins and aztreonam, but not cephamycins or carbapenems. $\beta$-lactamase inhi- bitors such as, clavulanic acid, sulbactam, and tazobactam usually inhibit them. ${ }^{4}$ Most ESBLs are derived from broadspectrum $\beta$-lactamases TEM-1 and SHV-1. Mutations of these genes result in alteration of the amino acid configuration around the active site of $\beta$-lactamases. ${ }^{4}$ Genes for ESBL are frequently encoded by plasmids. ${ }^{1}$

ESBL-producing organisms were first described in Europe. ${ }^{5}$ In the U.S., the first cases of ESBL organisms were reported in the $1988,{ }^{6}$ and the incidence since. ${ }^{7}$ A recent systematic review and meta-analysis have estimated a pooled prevalence of fecal colonization with ESBLs in healthy adults and children at $14 \%$ globally, with a higher prevalence of $22 \%$ in Southeast Asia and Africa. ${ }^{8}$ Number of studies from South and Southeast Asian countries have also demonstrated a likewise prevalence in food, food producing animals, and environment. ${ }^{8-12}$ Also, high colonization rates with ESBL-producing Enterobacteriaceae were found in travelers returning from South Asia. ${ }^{13-16}$ Multiple countries report emergence of community-associated infections with ESBL-producing E. coli. ${ }^{17-19}$ In the U.S., a recent prospective observational study showed an increase in the prevalence of ESBL-producing organisms, with $36.8 \%$ of all ESBL infections, caused by community-acquired ESBL producers. ${ }^{20} \mathrm{~A}$ 2013 report of the Centers for Disease Control and Prevention, classified ESBL-producing Enterobacteriaceae as a serious threat requiring prompt and sustained action.

ESBL-producing organisms have been reported to cause neonatal sepsis in other parts of the world with heterogeneity among the geographical location. ${ }^{3}$ ESBL infections in neonates have higher mortality rates compared with other pediatric populations.,21 The drug of choice for treatment of infections caused by ESBL-producing organisms is carbapenem. Among aminoglycosides, amikacin has the most activity against ESBLproducing strains and can be used if the organism is susceptible. Cefepime can also be used if the organism is susceptible. ${ }^{22}$

We observed 3 cases within a 6-month period, in 2017. All infants were born to families of South and South East Asian descent. These cases were managed with $\beta$-lactams and aminoglycosides without improvement. In our third case, early use of meropenem led to clinical improvement and survival. Infants with suspected sepsis, and whose mothers are from South and Southeast Asia, may have increased risk of infection with ESBL-producing organisms. Practitioners should consider adding meropenem to the initial antibiotic regimen in this patient population.

\section{Funding Source}

No funding was secured for this study.

\section{Financial Disclosure}

The authors have no financial relationships relevant to this article to disclose.

Conflict of Interest

The authors have no conflicts of interest to disclose.

Contributors' Statements

Authors K.D., T.L.S., and H.W. cared for the patient presented, reviewed the literature, and drafted the initial 
manuscript. Authors C.V.L., J.B.P., and L.W. cared for the patient, presented, reviewed, and revised the manuscript. All authors approved the final manuscript as submitted and agreed to be accountable for all aspects of the work.

\section{References}

1 Paterson DL, Bonomo RA. Extended-spectrum beta-lactamases: a clinical update. Clin Microbiol Rev 2005;18(04):657-686

2 Bush K, Jacoby GA. Updated functional classification of betalactamases. Antimicrob Agents Chemother 2010;54(03):969-976

3 Flokas ME, Karanika S, Alevizakos M, Mylonakis E. Prevalence of ESBL-producing Enterobacteriaceae in pediatric bloodstream infections: a systematic review and meta-analysis. PLoS One 2017;12(01):e0171216

4 Bradford PA. Extended-spectrum beta-lactamases in the 21st century: characterization, epidemiology, and detection of this important resistance threat. Clin Microbiol Rev 2001;14(04):933-951

5 Kliebe C, Nies BA, Meyer JF, Tolxdorff-Neutzling RM, Wiedemann B. Evolution of plasmid-coded resistance to broad-spectrum cephalosporins. Antimicrob Agents Chemother 1985;28(02):302-307

6 Jacoby GA, Medeiros AA, O'Brien TF, Pinto ME, Jiang H. Broadspectrum, transmissible beta-lactamases. N Engl J Med 1988;319 (11):723-724

7 Logan LK, Braykov NP, Weinstein RA, Laxminarayan R, Program CDCEP; CDC Epicenters Prevention Program. Extended-spectrum $\beta$-lactamase-producing and third-generation cephalosporin-resistant Enterobacteriaceae in children: trends in the United States, 1999-2011. J Pediatric Infect Dis Soc 2014;3 (04):320-328

8 Karanika S, Karantanos T, Arvanitis M, Grigoras C, Mylonakis E. Fecal colonization with extended-spectrum beta-lactamase-producing Enterobacteriaceae and risk factors among healthy individuals: a systematic review and metaanalysis. Clin Infect Dis 2016;63(03):310-318

9 Nguyen VT, Carrique-Mas JJ, Ngo TH, et al. Prevalence and risk factors for carriage of antimicrobial-resistant Escherichia coli on household and small-scale chicken farms in the Mekong Delta of Vietnam. J Antimicrob Chemother 2015;70(07):2144-2152

10 Jiang HX, Tang D, Liu YH, et al. Prevalence and characteristics of $\beta$ lactamase and plasmid-mediated quinolone resistance genes in Escherichia coli isolated from farmed fish in China. J Antimicrob Chemother 2012;67(10):2350-2353

11 Chen PA, Hung CH, Huang PC, et al. Characteristics of CTX-M extended-spectrum $\beta$-lactamase-producing Escherichia coli strains isolated from multiple rivers in southern Taiwan. Appl Environ Microbiol 2016;82(06):1889-1897

12 Bajaj P, Kanaujia PK, Singh NS, Sharma S, Kumar S, Virdi JS. Quinolone co-resistance in ESBL- or AmpC-producing Escherichia coli from an Indian urban aquatic environment and their public health implications. Environ Sci Pollut Res Int 2016;23(02):1954-1959

13 Kuenzli E, Jaeger VK, Frei R, et al. High colonization rates of extended-spectrum $\beta$-lactamase (ESBL)-producing Escherichia coli in Swiss travellers to South Asia-a prospective observational multicentre cohort study looking at epidemiology, microbiology and risk factors. BMC Infect Dis 2014;14:528

14 Ostholm-Balkhed A, Tärnberg M, Nilsson M, Nilsson LE, Hanberger H, Hällgren A; Travel Study Group of Southeast Sweden. Travel-associated faecal colonization with ESBL-producing Enterobacteriaceae: incidence and risk factors. J Antimicrob Chemother 2013;68(09): 2144-2153

15 Lübbert C, Straube L, Stein C, et al. Colonization with extendedspectrum beta-lactamase-producing and carbapenemase-producing Enterobacteriaceae in international travelers returning to Germany. Int J Med Microbiol 2015;305(01):148-156

16 Barreto Miranda I, Ignatius R, Pfüller R, et al. High carriage rate of ESBL-producing Enterobacteriaceae at presentation and followup among travellers with gastrointestinal complaints returning from India and Southeast Asia. J Travel Med 2016;23(02):tav024

17 Ben-Ami R, Rodríguez-Baño J, Arslan $\mathrm{H}$, et al. A multinational survey of risk factors for infection with extended-spectrum betalactamase-producing Enterobacteriaceae in nonhospitalized patients. Clin Infect Dis 2009;49(05):682-690

18 Pitout JD, Gregson DB, Church DL, Elsayed S, Laupland KB. Community-wide outbreaks of clonally related CTX-M-14 beta-lactamase-producing Escherichia coli strains in the Calgary health region. J Clin Microbiol 2005;43(06):2844-2849

19 Rodríguez-Baño J, Navarro MD, Romero L, et al. Epidemiology and clinical features of infections caused by extended-spectrum betalactamase-producing Escherichia coli in nonhospitalized patients. J Clin Microbiol 2004;42(03):1089-1094

20 Doi Y, Park YS, Rivera JI, et al. Community-associated extendedspectrum $\beta$-lactamase-producing Escherichia coli infection in the United States. Clin Infect Dis 2013;56(05):641-648

21 Sehgal R, Gaind R, Chellani H, Agarwal P. Extended-spectrum beta lactamase-producing gram-negative bacteria: clinical profile and outcome in a neonatal intensive care unit. Ann Trop Paediatr 2007;27(01):45-54

22 Kimberlin DW, Brady MT, Jackson MA, eds. Red Book 2018-2021: Report of the Committee on Infectious Diseases, 31st ed. Elk Grove Village, IL: American Academy of Pediatrics; 2018 